\title{
Gender Differences in Serum Prolactin Levels in Drug Naïve First Episode Schizophrenia
}

\author{
Amresh Shrivastava1, Nilesh Shah ${ }^{2}$, Avinash De Sousa², Sushma Sonavane ${ }^{2}$ \\ ${ }^{1}$ Department of Psychiatry, University of Western Ontario, Lawson Health Research Centre, London, Canada \\ ${ }^{2}$ Department of Psychiatry, Lokmanya Tilak Municipal Medical College, Mumbai, India \\ Email: avinashdes888@gmail.com
}

Received 25 February 2015; accepted 22 March 2015; published 27 March 2015

Copyright (C) 2015 by authors and Scientific Research Publishing Inc.

This work is licensed under the Creative Commons Attribution International License (CC BY).

http://creativecommons.org/licenses/by/4.0/

c) (i) Open Access

\begin{abstract}
Objective: To determine if there is a significant rise in serum prolactin (PRL) in acute psychosis related to disease process and weather there is a significant gender difference in the level of PRL and its correlation psychopathology. Procedure: Naturalistic study involved 60 consenting outpatients and inpatients in a tertiary psychiatric setting. Patient base was comprised of men $(n=30)$ and women $(n=30)$ with $83 \%$ in the age range of 21 - 40 years old. All subjects had confirmed first episode diagnosis set forth by the Diagnostic and Statistical Manual of Mental Disorders. Patients were drug free and drug naïve. Standardization for control was done using 15 control PRL samples obtained from healthy individuals. Methodology involved assessment by radioimmunoassay, the Brief Psychiatric Rating Scale and the Positive and Negative Syndrome Scale at day 1, 3 weeks and again at 6 weeks. All patients were treated with atypical antipsychotic drugs. Results: Serum PRL was elevated on baseline in $\mathbf{7 5 \%}$ of first episode psychosis schizophrenia, yet found to have a negative correlation to psychopathology at base week 3 and week 6 samples. Conclusions: Elevated serum PRL is not a potential indicator of acute schizophrenia. Levels are higher in females than in males, yet females respond better to treatment at a lower dose than males.
\end{abstract}

\section{Keywords}

Psychoendocrinology, Hyperprolactinemia, Dopaminergic System, Prolactin

\section{Introduction}

Prolactin (PRL) is a pituitary hormone that historically has an intimate relationship with schizophrenia as well as with common pharmacological treatments for schizophrenic patients. It is hypothesized that an increased dopa- 
minergic system would have a lowering effect on PRL levels; however, the opposite has been observed in clinical studies. This holds especially true in the drug naïve population of male patients. This contradiction warrants further study into the area of serum PRL and how it corresponds in drug naïve first episode schizophrenia with respect to gender differentiation.

Prolactin plays a vital role in proper functioning of the reproductive system [1]. Prolactin in excess can have effects on fertility in women and sexual function in men [2] which in turn can have a profound effect on an individual's level of self esteem and self worth. Such an outcome requires the clinician to take into account the consequences, this may hold in the long term. Hyperprolactinemia over a long duration has serious negative results in the overall health of the patient. This also applies to the drugs chosen to treat patients. Though atypical antipsychotic agents have a lesser incidence for elevating PRL levels, an increase is still seen. This increase contributes to negative sexual side effects and quality of life which has a large impact on patient compliance [3].

A look at serum PRL levels in drug naïve first generation schizophrenic patients offers an opportunity to note any correlation between prolactin levels and psychopathology [4]. Taking this a step farther and breaking the topic down additionally into gender comparison offers a more focused look at the subject matter.

\section{Methodology}

\subsection{Patients}

This study focuses on acute schizophrenia and first episode psychosis. Psychoendocrinological factors have an influence on the onset and resolution of schizophrenia. Thorough evaluation of each patient was taken which included clinical signs and symptoms in the areas of abnormal cognition and affect, neurological soft signs (NSS), and neurodegenerative and neuro developmental symptoms. Family history was also taken under consideration. Acute schizophrenia often develops into a chronic state, further emphasizing the need for proper documentation of a patient's current condition.

Patients were chosen based on specific criteria. Subjects consisted of both inpatient and outpatient individuals who were diagnosed with acute and subacute schizophrenia as per the Diagnostic and Statistical Manual of Mental Disorders (DSM IV) [5]. All were first episode, drug free and drug naïve. The study originated with 88 participants, 28 of which dropped out for varying reasons. The 60 remaining individuals ranged in age from 19 45 years old with the mean being 32.1 (83\% in 21 - 40 range) and were divided into two groups. A male group $(n=30)$ with a median age of 32.7 years old, and a female group $(n=30)$ with a mean age of 31.4 years old. Cause for exclusion included individuals with a history of previous psychological illness, exposure to medication and chemical codependence as well as alcoholism. Additionally, individuals with obvious or previously diagnosed endocrinological abnormalities were excluded. The study used 15 healthy individuals to provide control PRL levels on day 1 . All study group participants were treated with atypical antipsychotic drugs.

\subsection{Clinical and Laboratory Methods}

Serum PRL was taken from all participants using radioimmunoassay (RIA). This evaluation took place on day 1 (baseline), at 3 weeks and again at 6 weeks. Baseline data collected on the control group showed serum PRL with a mean of $17.5 \mathrm{ng} / \mathrm{ml}$. Control sample PRL for the male group registered a mean of $17 \mathrm{ng} / \mathrm{ml}$ while the female group registered a mean of $18 \mathrm{ng} / \mathrm{ml}$. Baseline data on the study group collectively yielded a mean PRL of $46.2 \mathrm{ng} / \mathrm{ml}$, females $64 \mathrm{ng} / \mathrm{ml}$, males $32.4 \mathrm{ng} / \mathrm{ml}$. This is a $37.8 \%$ increase over the $17.5 \mathrm{ng} / \mathrm{ml}$ from the control group. A mean of $46.2 \mathrm{ng} / \mathrm{ml}$ is greater than two times the normal value (1). The percentage of patients showing elevated baseline PRL was 75\%.

The Brief Psychiatric Rating Scale (BPRS) [6] and the Positive and Negative Syndrome Scale (PANSS) [7] were used for psychiatric evaluation of the participants. This evaluation also took place on day 1 (baseline), at 3 weeks and again at 6 weeks. Baseline mean BPRS when factoring both male and female scores collectively totaled 19.5 (range 11 - 33). Baseline PANSS when factoring both male and female scores collectively totaled 85 (range 81 - 99). Positive symptom (PS) on day 1 was 29, negative symptom (NS) was 12 and general psychosis (GP) was 44.

\section{Results}

Again, the male study group baseline PRL was $32.4 \mathrm{ng} / \mathrm{ml}$. The baseline mean BPRS for the male group specifically was 19.2. This figure decreased steadily over the next couple evaluations. At 3 weeks the mean was 9.05 
and at 6 weeks it was 5.75. This reduction expressed as a percentage is $29.9 \%$. The female group baseline PRL was $64 \mathrm{ng} / \mathrm{ml} ; 50.6 \%$ greater than that of the male group. Consistent reduction was also noted in the female group over the next 6 weeks. The 3 week mean BPRS was 10.4 and at 6 weeks was 4.5 giving an overall reduction in the female group of $22.5 \%$.

At 3 weeks, the mean BPRS for the study groups combined dropped from 19.5 to 9.5. The PANSS also dropped, originally 85, at 3 weeks it was 63 with PS at 18, NS at 10 and GP at 35. These figures showed a decrease again when evaluated at 6 weeks. Group BPRS now was 5.3. Group PANSS dropped to 55 with PS at 15, NS at 8 and GP at 32. Represented as a percentage, BPRS reduced 72.8\% from baseline. PANSS reduced 35.2\% from baseline with corresponding reduction as follows: PS 35.2\%, NS 33.3\% and GP 27.2\%. Serum PRL conclusively did not appear to have a positive correlation with psychopathology (Table 1).

Patients in the above study were all treated with atypical antipsychotic drugs (APD). Atypical APD were introduced into clinical practice more than 35 years ago with the first being clozapine [4]. Shortly after, the hematological side effect of agranulocytosis prompted widespread cessation of its use. Today it is again a common drug therapy due to its success in patients with treatment-resistant schizophrenia. Though clozapine is generally not warranted for treatment in first episode patients, there are 2 studies which have documented doing just that and concluded that clozapine as first-line treatment had no advantage over the use of other, more commonly used treatments [8]-[10]. It is recommended only for use with the severely ill schizophrenic patient due to the above mentioned risk of agranulocytosis and the additional risk of seizure. Where use of clozapine is indicated, close monitoring through weekly complete blood count (CBC) is essential.

Atypical antipsychotics (APD) have a lower occurrence of increasing PRL in patients than with the use of conventional APD. It is also documented that an initial rise in PRL is noted in patients treated with neuroleptics and will sustain or continue to rise within the first 3 months, after which the PRL stabilizes at a level lower than the initial rise [11]. It is important to note that though the level is lower, this level is typically still a hyperprolactinemia and thus has negative effects notably over the long term.

A common first-line drug therapy is risperidone. In a study comparing the relationship to PRL in the common drugs clozapine and risperidone, PRL was assessed in 120 patients following a 7 day wash out period [12]. A male group $(n=30)$ and a female group $(n=30)$ were formed for both pharmaceuticals. In the clozapine male group, the mean PANSS total reduction equated 36.2\% (respective reductions: PS $=42.7 \%$, NS $=43 \%$, GP $=$ $28.3 \%$ ) while the female group saw a total mean PANSS reduction of $44.2 \%$ (respective reductions: PS $=41.2 \%$, NS $=45.8 \%, \mathrm{GP}=46.1 \%$ ). In the risperidol male group, a reduction of $42.9 \%$ (respective reductions: $\mathrm{PS}=$ $45.6 \%$, NS $=46.6 \%$, GP $=43.4 \%$ ) was appreciated while the female group noted decreasing total mean PANSS by $49.4 \%$ (respective reductions: PS $=52.9 \%$, NS $=54.8 \%$, GP $=42.5 \%$ ).

Among the males taking clozapine, PRL related side effects in the study groups were as follows: weight gain $36.6 \%$, galactorrhea $10 \%$, sexual difficulties $20 \%$ and loss of erection $26.6 \%$. Compare that with the females taking clozapine: weight gain $40 \%$, galactorrhea $26.6 \%$, sexual difficulties $20 \%$ and amenorrhea $30 \%$. Among the men taking risperidone, PRL related side effects in the study groups were as follows: weight gain $10 \%$, sexual difficulties $10 \%$, and loss of erection $10 \%$. Compare that with the females taking risperidone: weight gain $13.3 \%$, galactorrhea $6.6 \%$, sexual difficulties $10 \%$ and amenorrhea $20 \%$.

Table 1. Serum prolactin and psychopathology correlation.

\begin{tabular}{|c|c|c|c|c|c|c|}
\hline \multicolumn{7}{|c|}{ Correlations (r) Prolactin \& Psychopathology Statistical Values } \\
\hline & \multicolumn{2}{|c|}{ Day 1} & \multicolumn{2}{|c|}{6 Weeks } & \multicolumn{2}{|c|}{5 Years } \\
\hline & $\mathrm{r}$ & $\mathrm{p}$ & r & $\mathrm{p}$ & r & $\mathbf{p}$ \\
\hline mBPRS & -0.93 & $<0.01$ & -0.89 & $<0.01$ & 0.87 & $<0.001$ \\
\hline PANSS (T) & -0.94 & $<0.01$ & -0.83 & $<1.001$ & 0.73 & $<\mathbf{0 . 0 0 1}$ \\
\hline PS & -0.28 & NS & -0.44 & NS & 0.53 & NS \\
\hline NS & +0.28 & NS & +0.36 & NS & 0.38 & NS \\
\hline GP & -0.92 & $<0.01$ & $-\mathbf{0 . 8 7}$ & $<0.01$ & 0.78 & $<0.01$ \\
\hline
\end{tabular}


Unfortunately, serum PRL levels of the male group taking clozapine are incomplete for unknown reasons, making analysis of the above findings for that group irrelevant. However, the female group taking clozapine yields complete statistics and the mean base PRL was $16.5 \mathrm{ng} / \mathrm{ml}$. On day 60 the level had risen $47.2 \%$ to $34.9 \mathrm{ng} / \mathrm{ml}$. The male group taking risperidone had a base PRL of $14.2 \mathrm{ng} / \mathrm{ml}$. On day 60 this level rose $33.3 \%$ to $42.6 \mathrm{ng} / \mathrm{ml}$, and the female risperidone group had a base PRL of $17 \mathrm{ng} / \mathrm{ml}$. This level had risen to 76.4 (22.2\%) by day 60 .

\section{Discussion}

Affecting 1\% of the population worldwide [12], serum PRL has a long documented intimate relationship with schizophrenia. Prolactin levels at base statistically seem to be more elevated in females than in males [13]. Additionally, higher quantities of female samples show elevation in PRL than in males. It is worth noting that though this may be the case, females tend to respond more favorably to treatment while maintaining a lower neuroleptic dose requirement than males [14]. Side effects associated with PRL are suffered comparably between the two groups.

In this study, it was observed that serum PRL was elevated in 75\% FES schizophrenia patients with $67 \%$ in the male population and $83 \%$ in the female. It was also concluded that baseline serum PRL had a negative correlation with respect to psychopathology at the base, week 3 and week 6 . However, there is one study indicating PRL as potentially aiding in determining schizophrenia subtypes. According to the study findings, low prolactin values seem to correspond with the paranoid type of schizophrenia, moderate with the schizoaffective and high for disorganized schizophrenia [15]. Even with this information, there are many other factors contributing to elevated PRL (medical and lifestyle) that must be examined and taken into consideration.

Despite the expected hyperprolactinemia in schizophrenic patients and with the use of neuroleptics, serum PRL levels in excess of $100 \mathrm{ng} / \mathrm{ml}$ should be investigated further in search for a prolactinoma [16].

\section{Conclusion}

This study concludes there is a negative correlation between serum PRL measured by RIA. Prolactin is statistically higher in females than in males and females seem to suffer hyperprolcatinemia in higher numbers. Careful consideration when choosing treatment will aid in mediating PRL related side effects and aid in compliance.

\section{Conflict of Interest}

Nil.

\section{References}

[1] Srerri, O., Chik, C.L., Ur, E. and Ezzat, S. (2003) Diagnosis and Management of Hyperprolactemia. CMAJ, 169, 575-581.

[2] Ghadirian, A.M., Chouinard, G. and Annable, L. (1982) Sexual Dysfunction and Plasma Prolactin Levels in NeurolepticTreated Schizophrenic Outpatients. Journal of Nervous \& Mental Disease, 170, 463-467. http://dx.doi.org/10.1097/00005053-198208000-00004

[3] Kinon, B.J., Gilmore, J.A., Liu, H. and Halbreich, U.M. (2003) Prevalence of Hyperprolactinemia in Schizophrenic Patients Treated with Conventional Antipsychotic Medications or Risperidone. Psychoneuroendocrinology, 28, 55-68. http://dx.doi.org/10.1016/S0306-4530(02)00127-0

[4] Wickipedia (2006) Antipsychotic Drugs. http://en.wikipedia.org/wiki/Atypical_antipsychotic

[5] American Psychiatric Association (2000) Diagnostic and Statistical Manual for the Classification of Psychiatric Disorders. American Psychiatric Publishing, New York.

[6] Overall, J.E. and Gorham, D.R. (1988) The Brief Psychiatric Rating Scale (BPRS): Recent Developments in Ascertainment and Scaling. Psychopharmacology Bulletin, 24, 97-99.

[7] Leucht, S., Kane, J.M., Kissling, W., Hamann, J., Etschel, E. and Engel, R.R. (2005) What Does the PANSS Mean? Schizophrenia Research, 79, 231-238. http://dx.doi.org/10.1016/j.schres.2005.04.008

[8] Robinson, D.G., Woerner, M.G., Delman, H.M. and Kane, J.M. (2005) Pharmacological Treatments for First-Episode Schizophrenia. Schizophrenia Bulletin, 31, 705-722. http://dx.doi.org/10.1093/schbul/sbi032

[9] Lieberman, J.A., Phillips, M., Gu, H., Stroup, S., Zhang, P., Kong, L., et al. (2003) Atypical and Conventional Antipsychotic Drugs in Treatment-Naïve First-Episode Schizophrenia: A 52-Week Randomized Trial of Clozapine vs Chlorpromazine. Neuropsychopharmacology, 28, 995-1003. 
[10] Woerner, M.G., Robinson, D.G., Alvir, J.M.J., Sheitman, B.B., Lieberman, J.A. and Kane, J.M. (2003) Clozapine as a First Treatment for Schizophrenia. The American Journal of Psychiatry, 160, 1514-1516. http://dx.doi.org/10.1176/appi.ajp.160.8.1514

[11] Brown, W.A. and Laughren, T.P. (1981) Tolerance to the Prolactin-Elevating Effect of Neuroleptics. Psychiatry Research, 5, 317-322. http://dx.doi.org/10.1016/0165-1781(81)90078-0

[12] Srivastava, A., Tamhane, M. and Kathrani, C.M. (1998) Serum Prolactin and Atypical Neuroleptics in Schizophrenia. Silver Mind Hospital, Mumbai.

[13] Melkersson, K.I., Hulting, A.-L. and Rane, A.J. (2001) Dose Requirement and Prolactin Elevation of Antipsychotics in Male and Female Patients with Schizophrenia or Related Psychoses. British Journal of Clinical Pharmacology, 51, 317- 324. http://dx.doi.org/10.1046/j.1365-2125.2001.01352.x

[14] Canuso, C.M., Goldstein, J.M. and Green, A.I. (1998) The Evaluation of Women with Schizophrenia. Psychopharmacology Bulletin, 34, 271-277.

[15] Segal, M., Avital, A., Rojas, M., Hausvater, N., Sandbank, S., Liba, D., et al. (2004) Serum Prolactin Levels in Unmedicated First-Episode and Recurrent Schizophrenia Patients: A Possible Marker for the Disease's Subtypes. Psychiatry Research, 127, 227-235. http://dx.doi.org/10.1016/j.psychres.2004.01.010

[16] Maas, D.L., Hunt, S., Mark, L. and Drobny, E.C. (1996) Reversable Thioridazine-Induced Magnetic Resonance ImagingDocumented Pituitary Enlargement Associated with Hyperprolactinemia. Endocrine Practice, 2, 85-89. http://dx.doi.org/10.4158/EP.2.2.85 